\title{
Zur Wahrnehmung von «Recht» und «Ethik» am Beispiel von Medizin-Studierenden
}

\author{
In der klinischen Praxis kommt es oft zu einer Vermischung aus rechtlichen und ethi- \\ schen Fragen, doch sie beziehen sich auf unterschiedliche Bereiche, und es sind un- \\ terschiedliche Fragestellungen. Im Studium braucht es Ausbildung in Ethik und Recht.
}

Rouven Porz

Die im Text dargestellte Meinung entspricht der des Autors, nicht notwendigerweise derjenigen der involvierten Institutionen.
Korrespondenz:

Dr. phil., dipl. biol. Rouven Porz, AdL

Inselspital

Universitätsspital Bern

CH-3010 Bern

Tel. 0316321956

rouven.porz[at]insel.ch
Beginnen wir direkt mit einem Beispiel zum straflosen Schwangerschaftsabbruch: Nur weil es in unserem Rechtssystem juristisch legitimiert ist, z. B. als Gynäkologin einen Schwangerschaftsabbruch durchführen zu dürfen (juristischer Kontext: «Fristenregelung» oder «Indikationsregelung»), so heisst dies noch lange nicht, dass diese Ärztin den Abbruch auch moralisch gut finden muss. Eine juristische Legitimation ist etwas anderes als eine moralisch-ethische Begründung. Ich halte dies für eine wichtige konzeptuelle Unterscheidung, insbesondere für Studierende der Medizin, d.h. für «Neulinge» in unserem Gesundheitssystem.

Mit dieser Unterscheidung zwischen dürfen und sollen kann man die Grenzen (und damit gleichzeitig auch die Verflechtungen!) zwischen Recht und Ethik recht gut illustrieren. Leider werden diese Grenzen und Verflechtungen in den Diskussionen um unser Gesundheitssystem aber kaum präzise thematisiert. Und dies, obwohl zurzeit sehr viel über Ethik in der Medizin geredet wird. Es bleibt weiterhin oft unklar, was gar unter Ethik verstanden werden kann, was Ethik leisten kann (in Abgrenzung zum Recht!) und welche Erwartungen an die zunehmend institutionalisierte Disziplin der Ethik überhaupt geknüpft sind. Ebendiese Unklarheiten schlagen sich - meiner Meinung nach - auch im Ethikunterricht an Schulen, Fachhochschulen und Universitäten nieder. Es besteht zwar scheinbar Einigkeit darin, dass unsere zukünftigen Health Care Professionals expliziter in Ethik ausgebildet werden müssen, aber die Ziele dieser Ausbildung werden nicht immer ebenso explizit benannt. Ich werde im Folgenden versuchen, mein eigenes Verständnis von Ethik zu explizieren, und ausserdem auf den Bedarf nach mehr juristischer Ausbildung im Medizinstudium hinweisen.

Ziele von Ethikunterricht für Medizinstudenten Was genau bedeutet eine Ausbildung in Ethik? Sollen die Studierenden ethische Kompetenzen aufbauen, Methoden erlernen, oder Haltungen entwickeln? Meist geht man von einem - wie ich finde eher unklaren - Portfolio dieser drei Bereiche aus, und vor allem werden die Studierenden auch kaum darin geschult, ethische Probleme trennscharf von recht- lichen, psychologischen oder rein medizinischen Zusammenhängen zu unterscheiden. Natürlich kann man hier einwenden, dass ethische Probleme meist gerade deshalb ethische Probleme sind, weil sie oft in einem komplexen Netzwerk aus medizinischen Fragen, rechtlichen Gegebenheiten und sozial-psychologischen Schwierigkeiten daherkommen. Klar, das mag teilweise stimmen, aber die genuin ethische Aufgabe ist es dann, möglichst präzise und analytisch das ethische Problem (bzw. die ethischen Probleme) herauszufiltern, zu reflektieren und zu analysieren, worin eigentlich der Wertekonflikt in diesem komplexen Netzwerk besteht. Ethik handelt von Werten. Die Ethik als Disziplin stellt die Frage nach dem «guten Handeln» - und welche Werte diesem Handeln zugrunde liegen. Dies gilt insbesondere für die klinische Ethik, für den Ethiksupport von Pflegenden, Ärzten usw. im praktischen Alltag [1]. Das heisst, klinische Ethik reflektiert Regeln und Wertvorstellungen von Berufsrollen, und dies insbesondere als Hilfestellung in Situationen, in denen das scheinbar alltägliche Handeln moralisch problematisch geworden ist. Ethik kann aber z.B. keine strafrechtlichen Sanktionierungen anordnen, wenn verbindliche Regeln verletzt worden sind. Dafür brauchen wir das Recht.

\section{Vermischung aus rechtlichen und ethischen Fragen}

Ich selbst verwende - z. B. im Unterricht bei Medizinstudierenden - sehr viel Zeit darauf, zwischen medizinischen, rechtlichen und ethischen Kompetenzen zu unterscheiden. Natürlich ist diese Unterscheidung insofern heuristisch, bzw. didaktisch, als dass die zukünftige Ärztin diese Dreiteilung in einem akuten klinischen Problemfall in professioneller Haltung immer zusammengefasst abrufen können muss. Dennoch lohnt es sich, davon bin ich pädagogisch überzeugt, gerade Studierenden deutlicher die Grenzen zwischen medizinischer, ethischer und rechtlicher Kompetenz aufzuzeigen. Nur über das erstmalige Aufzeigen der Abgrenzungen ist es später möglich, die Verflechtungen, Überlappungen und Gemeinsamkeiten zwischen Ethik und Recht in einem zweiten Schritt besser zu verstehen. 


\section{Was ist ein «ethischer Fall»?}

Bestätigt fand ich meine pädagogische Sichtweise auch in meiner Mitarbeit in einer wissenschaftlichen Auswertung der sogenannten «Ethikarbeiten 2010» an der Medizinischen Fakultät der Universität Bern [2]. Die Medizinstudierenden wurden vor ihrer ersten klinischen Erfahrung, vor ihren sogenannten «Blockpraktika» [3], aufgefordert, sich mit einem selbsterlebten ethischen Fall dieser ersten klinischen Erfahrungszeit in strukturierter Weise auseinanderzusetzen. Dazu hatte man ihnen als gedankliches Hilfsinstrument eine Zusammenfassung der vier medizinethischen Prinzipien (vom Grundgedanken her auf Beauchamp und Childress aufbauend) [4] vermittelt. Interessanterweise - so wissen wir heute, nach unserer Auswertung - wurde den Studierenden aber nicht (oder scheinbar nicht ausreichend genug) vermittelt, was überhaupt ein «ethischer» Fall sein könnte und was nicht. Die von den Studierenden zu schreibende strukturierte Ethikarbeit gliederte sich in eine Fallbeschreibung, eine ethische Fragestellung, das Aufzeigen von Lösungsvarianten. In unserer wissenschaftlichen Auswertung dieser Ethikarbeiten [5] wurde schnell deutlich, dass es den Medizinstudierenden aber kaum möglich war, überhaupt eine präzise ethische Fragestellung zu formulieren. Rückblickend gesprochen, aus professionell-ethischer Sicht, scheint dies ein augenfälliges Ergebnis zu sein: Wie soll man als ethischer Laie eine «ethische» Fragestellung formulieren, wenn einem gar nicht klar ist, was man unter Ethik überhaupt verstehen soll? Und ausserdem ist die Präzisierung einer ethischen Frage gerade in der Medizin oftmals so komplex, dass die «ethische Frage» in manchen ethischen Herangehensweisen und Fallbesprechungsmethoden erst am Ende der Fallbesprechung steht, sozusagen als Lösung des Reflexionsprozesses, und nicht am Anfang der Überlegungen.

Es bleibt $\mathrm{zu}$ folgern, dass man die Aufgabenstellung für die Medizinstudierenden präziser hätte formulieren können. Man hätte erklären müssen, dass ethische Fragestellungen immer auf Wertekonflikten beruhen, dass sie gemeinhin durch ein ungutes Bauchgefühl begleitet werden, und dass es in der Ethik gerade darum geht, nicht bei diesem Bauchgefühl stehen zu bleiben, sondern es zu versprachlichen, sich dann zu distanzieren, um eine transparente und begründbare Entscheidung fällen $\mathrm{zu}$ können. Und diese Begründung beruft sich auf einen Wert, den man in der angestrebten Handlung als eminent wichtig erachtet (und dies durchaus auch auf Kosten eines möglichen Schuldgefühls, das nach Wertekonflikten ein kaum zu vermeidender Beigeschmack einer ethischen Entscheidung sein kann).

\section{Eher rechtliche Fragen}

Für den vorliegenden Text möchte ich mich abschliessend aber noch einmal meiner Ausgangsüber- legung widmen, d.h. dem scheinbar unklaren $\mathrm{Zu}$ sammenhang zwischen Ethik und Recht.

\section{«Eine juristische Legitimation ist etwas anderes als eine moralisch- ethische Begründung.»}

Diese Unklarheit kann ich am besten aufzeigen, indem ich mich wieder auf einen weiteren Teil unserer Auswertungen beziehe, und zwar auf die Ergebnisse zur Doppel-Frage: In welcher Art von Blockpraktika nehmen die Studierenden überhaupt die meisten ethischen Probleme wahr? Und welcher Art sind diese ethischen Probleme? In unserer Auswertung zeigte sich als Ergebnis auf diese Fragen deutlich, dass die Psychiatrie eben gerade dieser meistgenannte Bereich war; konkret: freiheitsbeschränkende Massnahmen, Urteilsunfähigkeit, Umgangsweise mit psychischer Labilität und damit die juristische Grenze zwischen Selbst- und Fremdbestimmung. Für mich bestätigt dieses Ergebnis aber vor allem meine eingangs formulierte Forderung nach einer präziseren, trennscharfen und damit letztendlich verbindlicheren und verbindenden Ausbildung in Ethik und Recht. Der Schwerpunkt meiner Schlussfolgerung liegt jetzt auf dem Wörtchen und: Natürlich beinhalten alle diese zuletzt genannten Bereiche wie z.B. «freiheitsbeschränkende Massnahmen» grundlegende normative Überlegungen. Aber die Medizinstudierenden waren weniger an der grundlegenden Reflexion dieser Themen interessiert, sondern sie äusserten Unklarheiten darüber, was rechtlich erlaubt sei und was nicht. Zwei vereinfachte Beispiel in dieser Art: Darf ich die urteilsunfähige alleinstehende, 95-jährige Patientin operieren, wenn sie selbst nicht einwilligen kann? Oder: Darf ich die 32-jährige psychotische Patientin bei Fremdgefährdung in eine geschlossene Abteilung überweisen?

Dies sind - aus meiner Sicht - keine primär ethischen Fragen, sondern eher ein Appell an eine verbesserte juristische Ausbildung im Medizinstudium! Mit einer besseren rechtlichen Ausbildung wäre es dann auch leichter für die Studierenden, trennscharf die normative Reflexion zu vollziehen; also nicht nur die Frage aufzuwerfen, was man aus rechtlicher Sicht tun darf, ohne bestraft zu werden, sondern eben aus ethischer Sicht zu fragen, was man als Arzt tun soll, um eine problematische Handlung möglichst gut ausführen zu können. Gerade im Jahr der Einführung des neuen Kinder- und Erwachsenenschutzrechts scheint mir dies eine wichtige Schlussfolgerung: bitte mehr Rechtsausbildung im Medizinstudium, und dies gefordert von einem Ethiker! 